\title{
The effects of processing and mastication on almond lipid bioaccessibility using novel methods of in vitro digestion modelling and micro-structural analysis
}

\author{
Giuseppina Mandalari ${ }^{1,2}$, Myriam M.-L. Grundy ${ }^{3}$, Terri Grassby ${ }^{3}$, Mary L. Parker ${ }^{4}$, Kathryn L. Cross ${ }^{4}$, \\ Simona Chessa ${ }^{1}$, Carlo Bisignano ${ }^{5}$, Davide Barreca ${ }^{6}$, Ersilia Bellocco ${ }^{6}$, Giuseppina Laganà ${ }^{6}$, \\ Peter J. Butterworth ${ }^{3}$, Richard M. Faulks ${ }^{1}$, Peter J. Wilde ${ }^{7}$, Peter R. Ellis ${ }^{3}$ and Keith W. Waldron ${ }^{8_{*}}$ \\ ${ }^{1}$ The Model Gut, Institute of Food Research, Norwich NR4 7UA, UK \\ ${ }^{2}$ Dipartimento di Scienze del Farmaco e Prodotti per la Salute, University of Messina, Viale Annunziata, \\ 98168 Messina, Italy \\ ${ }^{3}$ Biopolymers Group, Diabetes and Nutritional Sciences Division, King's College London, Franklin-Wilkins Building, \\ 150 Stamford Street, London SE1 9NH, UK \\ ${ }^{4}$ Imaging Partnership, Institute of Food Research, Norwich NR4 7UA, UK \\ ${ }^{5}$ Dipartimento di Scienze Biologiche ed Ambientali, University of Messina, Salita Sperone 31, 98166 Messina, Italy \\ ${ }^{6}$ Dipartimento di Scienze Chimiche, University of Messina, Salita Sperone 31, 98166 Messina, Italy \\ ${ }^{7}$ Food Structure and Health Programme, Institute of Food Research, Norwich NR4 7UA, UK \\ ${ }^{8}$ The Biorefinery Centre, Institute of Food Research, Norwich NR4 7UA, UK \\ (Submitted 5 November 2013 - Final revision received 29 June 2014 - Accepted 13 July 2014 - First published online 15 September 2014 )
}

\begin{abstract}
A number of studies have demonstrated that consuming almonds increases satiety but does not result in weight gain, despite their high energy and lipid content. To understand the mechanism of almond digestion, in the present study, we investigated the bioaccessibility of lipids from masticated almonds during in vitro simulated human digestion, and determined the associated changes in cell-wall composition and cellular microstructure. The influence of processing on lipid release was assessed by using natural raw almonds (NA) and roasted almonds (RA). Masticated samples from four healthy adults (two females, two males) were exposed to a dynamic gastric model of digestion followed by simulated duodenal digestion. Between 7.8 and $11.1 \%$ of the total lipid was released as a result of mastication, with no significant differences between the NA and RA samples. Significant digestion occurred during the in vitro gastric phase (16.4 and $15.9 \%)$ and the in vitro duodenal phase $(32.2$ and $32.7 \%)$ for the NA and RA samples, respectively. Roasting produced a smaller average particle size distribution post-mastication; however, this was not significant in terms of lipid release. Light microscopy showed major changes that occurred in the distribution of lipid in all cells after the roasting process. Further changes were observed in the surface cells of almond fragments and in fractured cells after exposure to the duodenal environment. Almond cell walls prevented lipid release from intact cells, providing a mechanism for incomplete nutrient absorption in the gut. The composition of almond cell walls was not affected by processing or simulated digestion.
\end{abstract}

Key words: Almonds: Lipid bioaccessibility: Mastication: Cell walls: Processing

In recent clinical studies, almond consumption has been shown to have beneficial effects on blood glucose levels in individuals with type 2 diabetes and prediabetes, with significant reductions in fasting blood glucose, insulin, and LDL-cholesterol concentrations as well as improvements in glycaemic control and insulin resistance ${ }^{(1,2)}$. A number of studies have also demonstrated the paradox that despite the high fat content of almonds (typically in the range of 50-55\%), their consumption is not associated with higher body weight or $\mathrm{BMI}^{(3-5)}$. This may be attributed to a number of mechanisms, notably a reduction in nutrient digestion, increased satiety and/or increased energy compensation through reduced food intake ${ }^{(6,7)}$. Indeed, the effect of mastication of almonds on satiety and gut hormone response has

Abbreviations: CDTA, 2,2',2", $2^{\prime \prime \prime}-((1 R, 2 R)-1,2$-cyclohexanediyldinitrilo)tetraacetic acid; DGM, dynamic gastric model; NA, natural raw almonds; RA, roasted almonds.

*Corresponding author: K. W. Waldron, fax +44 1603 507723, email keith.waldron@ifr.ac.uk 
been investigated in a randomised cross-over feeding study involving thirteen healthy volunteers ${ }^{(8)}$. The results of the present study showed that after forty chews, hunger was significantly suppressed and satiety elevated above baseline for a longer period of time compared with twenty-five chews. These data demonstrated that mastication itself may have an effect on fat digestion and absorption in the gut, on hormone release, weight management and satiety. In addition, we have previously reported that a significant proportion of lipid and other macronutrients in almonds remain undigested in the gastrointestinal tract of healthy human volunteers, and that the structural integrity of almond cell walls (dietary fibre) plays an important role in regulating lipid bioaccessibility and subsequent digestion ${ }^{(9,10)}$. The importance of these findings has been highlighted by recent data showing the crucial role played by almond cell walls in regulating lipid bioaccessibility and postprandial lipaemia, an attenuation of which is associated with a reduced risk of $\mathrm{CVD}^{(11)}$. However, there is limited information concerning the relative amounts of lipid in almonds (or, indeed, other plant-derived foods) released from the cellular structure in different parts of the gastrointestinal tract during digestion, or the impact of mastication on lipid release.

To elucidate the temporal relationship between bioaccessibility and almond cell structure, we investigated lipid bioaccessibility from masticated almonds and the associated changes that occur in the structure and composition of cell walls during processing and digestion. In the present study, we defined bioaccessibility as the proportion of a nutrient that can be released from a complex food matrix and therefore becomes potentially available for digestion and/or absorption in the gastrointestinal tract. To assess the effect of processing on lipid release, raw and roasted almonds of the same variety were used. A dynamic gastric model of digestion (DGM) was used for the first time to facilitate improved mechanistic understanding of lipid digestion. The DGM provides a realistic and predictive simulation of physical and chemical processing and accurately mimics both the transit time and the luminal environment within the human stomach ${ }^{(12,13)}$. The DGM works in real time with the meal being processed as eaten. As in the human stomach, masticated material is processed in functionally distinct zones: within the fundus/main body of the DGM, where gastric acid and enzyme secretion are introduced at physiological secretion rates, and within the antrum where the food is subjected to physiological shear stress and grinding forces.

In our previous study ${ }^{(10)}$, we quantified lipid bioaccessibility in vitro in almonds using blocks of cotyledon tissue with defined geometry (i.e. $2 \mathrm{~mm}$ cubes). The changes in lipid distribution in the cells of these blocks during processing and digestion were investigated by microscopy of resin-embedded sections, which is time consuming for multiple samples. In the present study, we combined human mastication with the DGM and simulated duodenal digestion phases for the first time. The mastication process is a key stage in the physical trituration of food, and in the case of almonds, determines the nature and degree of tissue and cell fracture, and thus is likely to strongly influence lipid bioaccessibility. A preliminary investigation, using tissue blocks from our previous study, was first undertaken to assess the feasibility of using $2,2^{\prime}, 2^{\prime \prime}, 2^{\prime \prime \prime}$ $((1 R, 2 R)-1,2 \text {-cyclohexanediyldinitrilo)tetraacetic acid (CDTA })^{(14)}$ to separate the cells for microscopy examination, instead of the conventional sectioning method.

\section{Materials and methods}

\section{Almonds}

Food-grade natural raw almonds, the NA sample (Prunus dulcis Miller D.A. Webb; variety Nonpareil) used in the present study were produced by Hughson Nut and kindly provided by the Almond Board of California. The almonds were roasted (RA sample) by the Almond Board of California following a standardised method of hot air (dry) roasting $\left(150^{\circ} \mathrm{C}\right.$ for $15 \mathrm{~min}$ ). The almonds were stored at $4^{\circ} \mathrm{C}$ until required.

\section{Chemicals and enzymes}

Egg L- $\alpha$-phosphatidylcholine (lecithin grade 1, $99 \%$ purity) was obtained from Lipid Products. Porcine gastric mucosa pepsin (activity 3300 units/mg protein), bovine $\alpha$-chymotrypsin (activity 40 units/mg protein using N-benzoyl-L-tyrosine ethyl ester (BTEE) as the substrate), pancreatic $\alpha$-amylase (activity 10 units/mg solid using starch as the substrate) and porcine trypsin (activity 13800 units/mg protein using $\mathrm{N \alpha}$ benzoyl-L-arginine ethyl ester (BAEE) as the substrate) were obtained from Sigma-Aldrich Chemical Company. One unit of pepsin is defined as the amount to produce a $\Delta A_{280}$ of 0.001 per min at $\mathrm{pH} 2.0$ at $37^{\circ} \mathrm{C}$, measured as TCA-soluble products using $\mathrm{Hb}$ as the substrate. One unit of $\alpha$-chymotrypsin hydrolyses $1.0 \mu \mathrm{mol} \mathrm{BTEE} / \mathrm{min}$ at $\mathrm{pH} 7.8$ at $25^{\circ} \mathrm{C}$. One unit of pancreatic $\alpha$-amylase liberates $1.0 \mathrm{mg}$ maltose from starch in $3 \mathrm{~min}$ at $\mathrm{pH} 6.9$ at $20^{\circ} \mathrm{C}$. One unit of BAEE is defined as the amount to produce a $\Delta A_{253}$ of 0.001 per min at $\mathrm{pH} 7.6$ $\left(25^{\circ} \mathrm{C}\right)$ using BAEE as the substrate. Lipase for the gastric phase of digestion was a gastric lipase analogue of fungal origin (F-AP15, activity $>150$ units/mg) obtained from Amano Enzyme, Inc. One unit of activity is defined as the amount of enzyme that liberates the equivalent of $1 \mu \mathrm{mol}$ fatty acid/min from a substrate emulsion of olive oil at $\mathrm{pH}$ 7·0. Porcine pancreatic lipase (activity 25600 units/mg protein), porcine colipase and bile salts were obtained from SigmaAldrich. One unit of porcine pancreatic lipase hydrolyses 1.0 microequivalent of fatty acid from a TAG in $1 \mathrm{~h}$ at $\mathrm{pH} 7 \cdot 7$ at $37^{\circ} \mathrm{C}$ using olive oil.

\section{Almond mastication method}

A total of four healthy adults (two females, two males) participated in the mastication stage (mean age 22.0 (SEM 1.4) years and BMI $\left.24 \cdot 2(\operatorname{sem} 3 \cdot 3) \mathrm{kg} / \mathrm{m}^{2}\right)$. Exclusion criteria included the following: allergy to almonds or related allergens (other tree nuts, celery, pears, apples, cherries, peaches and parsley); incomplete dentition, other than unerupted wisdom teeth; any dental treatment in the last 3 months, except check-ups; current infectious disease. 
After a full explanation of the study aims and procedure, each participant signed a written consent. Each participant attended two mastication sessions, one per type of almond, namely raw (NA) and roasted (RA), and asked to masticate about $28 \mathrm{~g}$ of almonds, an amount which represents a standard portion. The participants self-divided the portion into mouthfuls, each of which was masticated until they felt the urge to swallow, at which stage they expectorated the contents of their mouth into a pre-weighed plastic container.

The number of mastication cycles ( $N$, counted cycles) and the mastication duration ( $T$, duration of sequences) were recorded and averaged. Mastication frequency was calculated by dividing $N$ by $T$. The volunteers then rinsed their mouth with about $30 \mathrm{~g}$ of water and to maximise recovery, emptied it into the container used previously. The addition of saliva during mastication further increased the weight of the recovered sample to approximately $75 \mathrm{~g}$. The present study was conducted according to the guidelines laid down in the Declaration of Helsinki, and all procedures involving human subjects were approved by the Research Ethic Committee of the North London's National Research Ethics Service (NRES), UK (no. 10/H0717/096). Written informed consent was obtained from all subjects.

\section{Simulated human digestion}

Gastric digestion. Individual masticated NA ( $n$ 4) or RA ( $n$ 4) samples $(60 \mathrm{~g})$ were fed onto the DGM for $60 \mathrm{~min}$ together with a representative drink of water $(150 \mathrm{ml})$ in the presence of priming acid $(20 \mathrm{ml})$, whose composition has been reported previously $^{(13)}$. The simulated gastric secretion, bile and pancreatic juice were prepared as reported previously ${ }^{(10,13)}$. The simulated gastric acid solution contained $0 \cdot 2 \mathrm{M}-\mathrm{HCl}, 0.08 \mathrm{M}$ $\mathrm{NaCl}, 0.03 \mathrm{~mm}_{-} \mathrm{CaCl}_{2}$, and $0.9 \mathrm{~mm}-\mathrm{NaH}_{2} \mathrm{PO}_{4}$. The simulated gastric enzyme solution was prepared by dissolving porcine gastric mucosa pepsin and a gastric lipase analogue from Rhizopus oryzae in the above-described salt mixture (no acid) at a final concentration of 9000 and 60 units $/ \mathrm{ml}$ for pepsin and lipase, respectively. A suspension of single-shelled lecithin liposomes, prepared as described previously ${ }^{(10)}$, was added to the gastric enzyme solution at a final concentration of $0.127 \mathrm{~mm}$. A total of six samples ( $48 \mathrm{~g}$ for each NA and RA sample) were removed from the DGM at $10 \mathrm{~min}$ intervals. The amounts of acid secretion added during gastric digestion were 17.4 (SD 3) and 18.7 (SD 2) $\mathrm{ml}$ for the NA and RA samples, respectively. The amounts of gastric enzymes added during gastric digestion were 19.0 (SD 3) and 21.0 (SD 4) $\mathrm{ml}$ for NA and RA, respectively. Each gastric sample was weighed, its $\mathrm{pH}$ recorded and adjusted to $7 \cdot 0$ with $1 \mathrm{M}-\mathrm{NaOH}$ to inhibit gastric enzyme activity.

Duodenal digestion. A pooled sample (42g), obtained from an aliquot $(7 \mathrm{~g})$ of each gastric sample, was transferred to a Sterilin plastic tube for duodenal digestion with the addition of simulated bile solution $(8.4 \mathrm{ml})$ and pancreatic enzyme solution $(23.5 \mathrm{ml})$, and incubated at $37^{\circ} \mathrm{C}$ under shaking conditions (170 rpm) for $2 \mathrm{~h}$. Simulated bile was prepared fresh daily. It contained $6.5 \mathrm{~mm}$-lecithin, $4 \mathrm{~mm}$-cholesterol, $12.5 \mathrm{~mm}$-sodium taurocholate and $12.5 \mathrm{~mm}$-sodium glycodeoxycholate in a solution containing $146.0 \mathrm{~mm}-\mathrm{NaCl}, 2.6 \mathrm{~mm}-\mathrm{CaCl}_{2}$ and $4.8 \mathrm{~mm}-\mathrm{KCl}$.

Pancreatic enzyme solution contained $125.0 \mathrm{~mm}-\mathrm{NaCl}$, $0.6 \mathrm{~mm}^{-\mathrm{CaCl}_{2}}, 0.3 \mathrm{~mm}-\mathrm{MgCl}_{2}$ and $4 \cdot 1 \mu \mathrm{m}-\mathrm{ZnSO}_{4} \cdot 7 \mathrm{H}_{2} \mathrm{O}$. Porcine pancreatic lipase $(590$ units $/ \mathrm{ml})$, porcine colipase $(3.2 \mu \mathrm{g} /$ $\mathrm{ml}$ ), porcine trypsin (11 units/ml), bovine $\alpha$-chymotrypsin (24 units $/ \mathrm{ml}$ ) and porcine $\alpha$-amylase (300 units $/ \mathrm{ml}$ ) were added to the pancreatic solution. Each gastric sample removed from the DGM every $10 \mathrm{~min}$ and the pooled duodenal sample after $2 \mathrm{~h}$ incubation were centrifuged at $3700 \mathrm{rpm}$ for $15 \mathrm{~min}$ $\left(7^{\circ} \mathrm{C}\right)$ to separate the soluble fraction from the residue. All samples were immediately snap-frozen in liquid $\mathrm{N}_{2}$ and retained for analyses.

\section{Particle size distribution}

Representative aliquots of the samples (NA, $n 4$ and RA, $n$ 4) obtained after mastication and DGM digestion were poured onto a sieve (1700 $\mu \mathrm{m}$ aperture), placed on top of a sieve base covered with a nylon mesh $(20 \mu \mathrm{m}$ mesh size) and washed with deionised water. Particles larger than $1700 \mu \mathrm{m}$ were too large for the laser diffraction instrument, whereas those smaller than $20 \mu \mathrm{m}$ were cell fragments and released nutrients and could therefore be discarded. The particles retained on the mesh were collected as an aqueous suspension and loaded into the Malvern Laser Diffraction Particle Sizer $2000^{\circledR}$ (Malvern Instruments Limited). Mie theory was selected for these measurements. Almond oil and water have a refractive index of 1.471 and 1.330 , respectively. The absorption of almond particles was $0 \cdot 1$. Each sample was divided into two to three approximately equal fractions and poured, one after the other, into the automated sample dispersion unit (Hydro 2000G) filled with water. For each fraction, ten consecutive measurements of $10 \mathrm{~s}$ duration were taken. The replicates were averaged to give the particle size distribution for the whole sample. Particle sizes were obtained as the volume percentage of the total volume of all particles present in the distribution. The means of the particle size distributions were calculated by the Malvern Laser Diffraction Particle Sizer 2000 software algorithm.

\section{Lipid content determination}

Original almond materials (NA and RA), post-mastication and digesta residues recovered were analysed for total lipid. Lipid extraction was performed using a Soxhlet extraction $\operatorname{method}^{(15)}$, with $n$-hexane as the solvent. The results of lipid content analysis are expressed as a percentage of dry weight.

\section{Cell-wall analysis}

To assess any changes in the cell-wall composition of almonds after mastication and digestion or through any effect of processing, cell-wall material was prepared from the following constituents using a modified method as described previously ${ }^{(9)}$ : (1) original almonds, NA (raw) and RA (roasted); (2) masticated and digested NA, after gastric digestion (NA G) and gastric + duodenal digestion (NA G+D); (3) masticated and digested RA, after gastric digestion (RA G) and gastric + duodenal 
digestion (RA G+D). Alditol acetates were quantified by GLC, using the method of Blakeney et al. ${ }^{(16)}$. Total uronic acids were determined colorimetrically at $580 \mathrm{~nm}$ following the method of Blumenkrantz \& Asboe-Hansen ${ }^{(17)}$.

\section{Microstructural analyses}

Microstructural analyses of processed and digested almonds have previously been carried out on resin-embedded sections $^{(9,10,14)}$. Owing to the dense nature and high lipid content of almond tissue, the penetration of fixatives, particularly lipid-stabilising osmium tetroxide, into all but the smallest samples is often suboptimal.

An alternative untested approach would be to first separate the almond cells in the raw, roasted and treated tissue using the Ca-chelating agent $\mathrm{CDTA}^{(14)}$, and then to examine these cells by bright-field or fluorescence microscopy to view directly their individual lipid content. To assess the feasibility of this novel approach, a preliminary experiment was carried out using well-defined $2 \mathrm{~mm}^{3}$ blocks of natural raw, roasted and natural raw $\mathrm{G}+\mathrm{D}$ almond tissue ${ }^{(10)}$, which were prepared using sharp razor blades to minimise tissue damage. Briefly, the almond blocks were placed into CDTA $\left(50 \mathrm{~mm}-\mathrm{Na}_{3} \mathrm{H}_{1-}\right.$ $\mathrm{CDTA}+5 \mathrm{~mm}-\mathrm{Na}_{2} \mathrm{~S}_{2} \mathrm{O}_{5}, \mathrm{pH}$ 7) for at least 4 weeks. For brightfield microscopy, the softened blocks were then gently pressed with a spatula in a drop of CDTA on a microscope slide to separate the cells. For fluorescence microscopy, the blocks were pressed in a drop of $0.01 \%$ Nile Blue (CI 51 180). The separated cells were examined and imaged using an Olympus BX60 microscope (Olympus), with ProgRes ${ }^{\circledR}$ Capture Pro 2.1 software (Jenoptik). To localise lipids stained with Nile Blue, the NB filter cube (U-MNB, exciter filter BP470-490, barrier filter (BA515)) of the microscope was used.

The results of this feasibility study showed that in CDTAseparated almond cells, lipid either as oleosomes, coalesced droplets or partially digested masses is readily identifiable by light microscopy, without the need for embedding or the use of hazardous chemicals such as osmium tetroxide (see the Results section). Therefore, microscopy analysis of 1-2 $\mathrm{mm}$ particles of masticated raw, roasted and digested almond tissue was undertaken using the same method.

\section{Statistical analysis}

ANOVA was used to test for differences in cell-wall composition and lipid release after the gastric and gastric-duodenal digestion phases, using SAS/SPSS software. Post hoc analysis using Tukey's honestly significant difference was used to examine each pairwise difference. To examine the differences between the raw and roasted almond samples, two sample $t$ tests (two-tailed) were used. All results are presented as means with their standard errors, unless otherwise stated.

\section{Results}

\section{Mastication}

Table 1 reports the mastication parameters for the NA and RA samples. A slightly higher number of mastication cycles and
Table 1. Mastication parameters for natural raw and roasted almonds (Mean values with their standard errors, $n 4$ )

\begin{tabular}{|c|c|c|c|c|c|c|}
\hline \multirow[b]{2}{*}{ Almond meals } & \multicolumn{2}{|c|}{$\begin{array}{l}\text { Number of } \\
\text { cycles }\end{array}$} & \multicolumn{2}{|c|}{$\begin{array}{l}\text { Sequence } \\
\text { duration (s) }\end{array}$} & \multicolumn{2}{|c|}{$\begin{array}{l}\text { Mastication } \\
\text { frequency } \\
\text { (per s) }\end{array}$} \\
\hline & Mean & SEM & Mean & SEM & Mean & SEM \\
\hline Natural almonds & 28.3 & 1.9 & $22 \cdot 9$ & $2 \cdot 7$ & $1 \cdot 26$ & $0 \cdot 1$ \\
\hline Roasted almonds & 24.9 & $2 \cdot 1$ & $19 \cdot 8$ & $2 \cdot 4$ & $1 \cdot 28$ & 0.1 \\
\hline
\end{tabular}

sequence duration were recorded for the NA sample compared with the RA sample, but no differences $(P=0.051$ and 0.059 , respectively) were found between the two almond meals. These basic parameters for mastication are expected to change with the individual as well as the food and its physical form. However, in the present study, since there were negligible differences in mastication parameters between the NA and RA samples, any effects of roasting on lipid release may not be linked to masticatory behaviour.

\section{Particle size distribution}

Fig. 1(a) and (b) shows the average particle size distribution of the NA and RA samples following mastication or mastication + in vitro gastric digestion. All the size distributions were multimodal and broad especially for the RA samples. Compared with masticated raw almonds, roasted almond boluses (chewed meals expectorated by the volunteers) are composed of particles of smaller size; thus, the mean particle size was 500 (SEM 29) and 365 (SEM 12) $\mu \mathrm{m}$ for the NA and RA samples, respectively. This was also shown by the difference in the maximum particle size: 1002 (SEM 0.2) $\mu \mathrm{m}$ for the NA sample and 893 (SEM 0.1) $\mu \mathrm{m}$ for the RA sample. No difference ( $P=0.262$ and 0.386 for the NA and RA samples, respectively) was found in the overall distribution between the masticated and post-gastric almond samples (mean particle size 498 (SEM 30) and 354 (sem 20) $\mu \mathrm{m}$ for the raw and roasted postgastric samples, respectively). The fact that the height and position of the main peaks are unchanged indicated that physical process of digestion did not lead to significant particle disintegration. The minor changes to the distribution observed between 10 and $100 \mu \mathrm{m}$ following digestion could be due to internal changes in the microstructure of the particles, such as oil-body coalescence or local changes in the refractive index. These changes are difficult to predict in particles with complex light-scattering properties.

\section{Total lipid loss during digestion}

The release of total lipid as a percentage of the original amount present $(54.5 \%, \mathrm{w} / \mathrm{w})$ for the NA and RA samples, after mastication, in vitro gastric and gastric + duodenal digestion, is shown in Fig. 2. Between $7 \cdot 8$ and $11.1 \%$ of the original lipid in the NA and RA samples, respectively, was released as a result of mastication, with no statistically significant differences between these samples. An increase in lipid release was observed during the duodenal phase $(32.2$ and $32.7 \%$ for the NA and RA samples, respectively) compared with that detected in the 

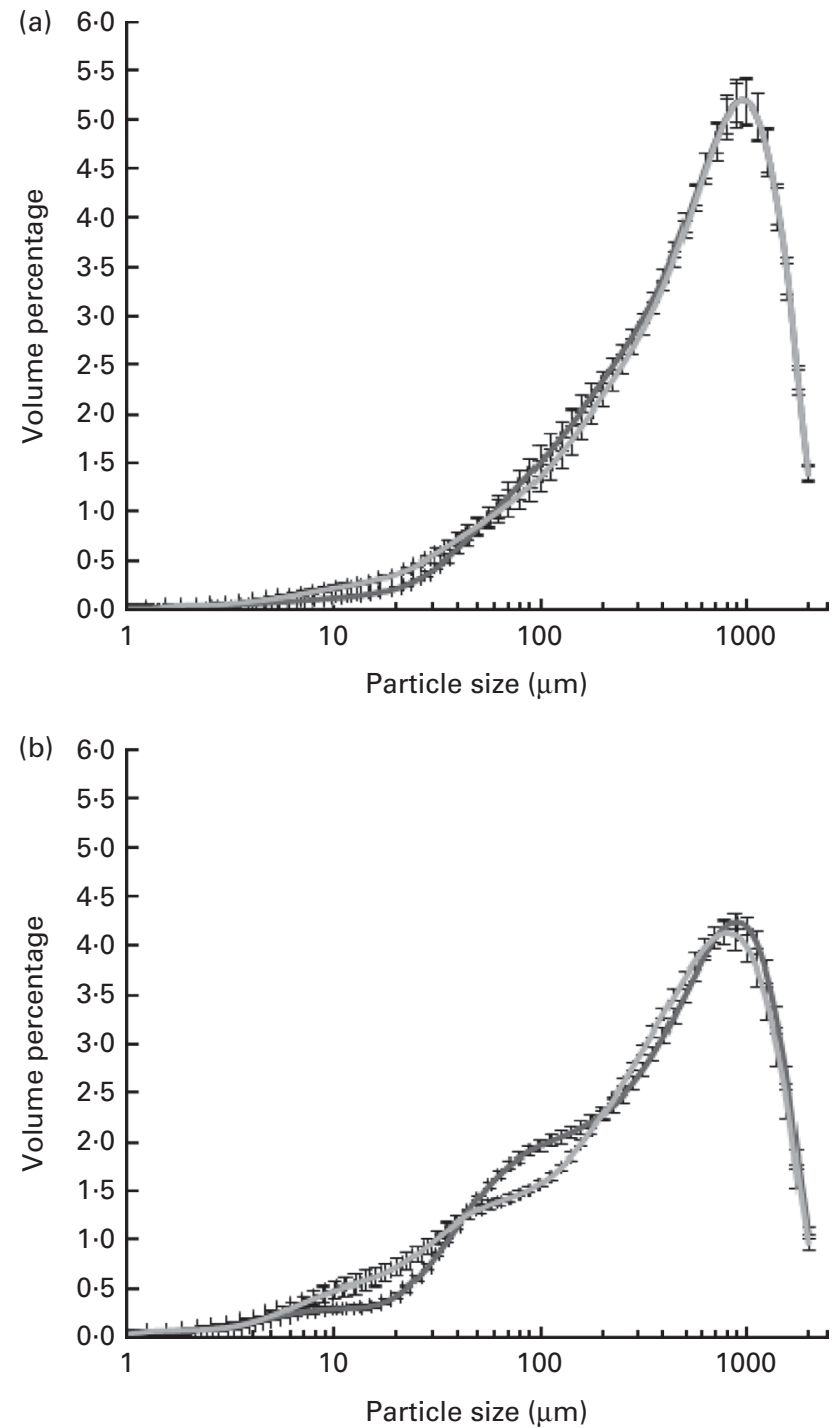

Fig. 1. Particle size distribution of raw (a) and roasted (b) almonds following mastication and in vitro gastric digestion. Values are means $(n 4)$, with standard errors represented by vertical bars. Mean values were significantly different in particle size at all size fractions found between the raw and roasted almonds (before digestion), except for sizes 141, 159, 178 and $200 \mu \mathrm{m}(P<0.001$; Student's paired $t$ test). - Chewed raw almonds; post-gastric raw almonds; - , chewed roasted almonds; - , post-gastric roasted almonds.

gastric environment. These findings differ considerably from our previous investigation that focused on sharp-cut almond cubes and finely ground almonds. In that study, gastric + duodenal digestion produced only a slight increase in lipid release over and above that of the gastric phase alone ${ }^{(10)}$.

No significant differences in lipid release were observed between the NA and RA samples after in vitro gastric and gastric + duodenal digestion.

\section{Effect of processing and digestion on the cell-wall composition of almonds}

The sugar composition of the cell-wall material of the original NA and RA samples and the same almond types after gastric and gastric + duodenal digestion are shown in Table 2 . Almond cell walls are mainly composed of arabinose-rich polysaccharides, including pectic substances, presumably encasing the cellulose microfibrils. As reported previously ${ }^{(9,10)}$, arabinose, glucose and galacturonic acid were the major sugars present in all samples tested, followed by xylose. Between 3 and $5 \%$ of the total sugar content was galactose in all samples, whereas smaller amounts (1-2\% of the total sugar content) of rhamnose and fucose were detected. No statistically significant differences in the major identified sugars (arabinose, glucose, galacturonic acid and xylose) were observed between the NA and RA samples after the gastric and gastric + duodenal phases, indicating that processing had no effect on the chemical composition of the cell walls before or after simulated digestion. An increase $(P=0.03)$ in galacturonic acid was detected in the RA samples that were processed by gastric + duodenal digestion compared with the original undigested samples; however, this effect was not found in the corresponding roasted samples.

\section{Effect of mastication and digestion on the microstructure of almond cotyledon}

Preliminary investigation using almond tissue blocks. This feasibility trial showed it was possible, using blocks of almond tissue softened in CDTA and bright-field or fluorescence microscopy, to recognise unequivocally the distribution of lipid within a large number of cells in each sample (Fig. 3). In the cells of NA, the fluorescent lipid stain Nile Blue (Fig. 3(a)) clearly showed an even distribution of lipid in all cells, which was also observed by bright-field microscopy (Fig. 3(b)). At high magnification (100X oil immersion objective), the lipid (Fig. 3(c)) was clearly located in spherical structures (mean size $2.5 \mu \mathrm{m}$ ) identifiable by their size and shape as lipid bodies or oleosomes ${ }^{(9,10)}$. In all cells from roasted almond blocks (Fig. 3(d) and (e)), the distribution of lipid was uneven due to heat-induced

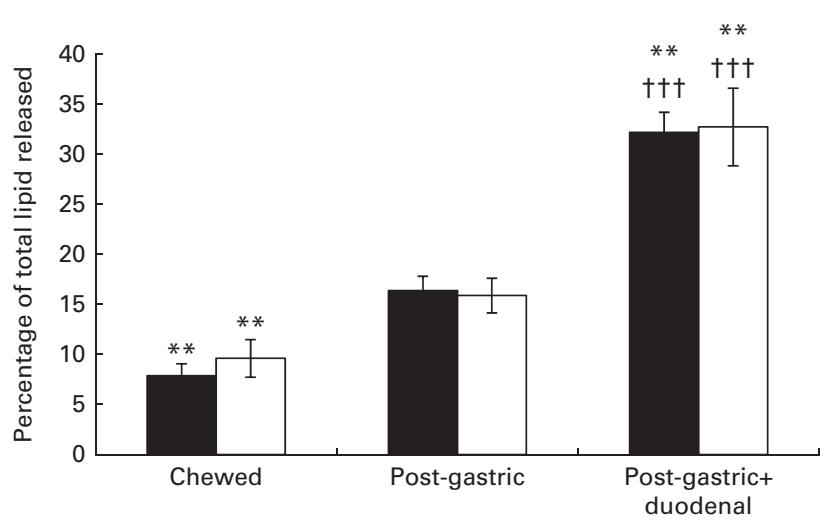

Fig. 2. Total lipid loss (\%) from natural raw ( $\square ; n$ 4) and roasted ( $\square ; n$ 4) almonds due to mastication, in vitro gastric and gastric + duodenal digestion. Values are means, with standard errors represented by vertical bars. Significant differences after the gastric and gastric-duodenal digestion phases were calculated by ANOVA $(P<0.005)$. ${ }^{* *}$ Mean values were significantly different from those for the post-gastric condition $(P<0.005)$. †††Mean values were significantly different from those for the chewed condition $(P<0.0005)$. 
Table 2. Sugar composition (mol \%) of natural raw almonds (NA), natural raw almonds after in vitro gastric digestion (NA G), natural raw almonds after in vitro gastric + duodenal digestion (NA G+D), roasted almonds $(R A)$, roasted almonds after in vitro gastric digestion (RA G), roasted almonds after in vitro gastric + duodenal digestion (RA G+D)

(Mean values of three replicates and standard deviations)

\begin{tabular}{|c|c|c|c|c|c|c|c|c|c|c|c|c|}
\hline \multirow[b]{2}{*}{ Sugar } & \multicolumn{2}{|c|}{ NA } & \multicolumn{2}{|c|}{$N A G$} & \multicolumn{2}{|c|}{$N A G+D$} & \multicolumn{2}{|c|}{ RA } & \multicolumn{2}{|c|}{ RA G } & \multicolumn{2}{|c|}{$R A G+D$} \\
\hline & Mean & SD & Mean & SD & Mean & SD & Mean & SD & Mean & SD & Mean & SD \\
\hline Rhamnose & 1.6 & 0.1 & 1.9 & $<0.1$ & 1.7 & $<0.1$ & 1.7 & 0.1 & $1 \cdot 7$ & 0.1 & $2 \cdot 0$ & $0 \cdot 1$ \\
\hline Fucose & 1.0 & $<0.1$ & 0.9 & $<0.1$ & 0.9 & $<0.1$ & 0.8 & $<0.1$ & 0.9 & $<0.1$ & 1.0 & $<0.1$ \\
\hline Arabinose & 37.3 & 1.4 & $32 \cdot 2$ & 0.6 & 31.3 & 1.0 & 31.1 & 0.7 & $33 \cdot 6$ & 0.8 & $32 \cdot 8$ & 0.5 \\
\hline Xylose & 11.6 & 0.5 & $9 \cdot 6$ & 0.1 & $9 \cdot 8$ & 0.3 & $10 \cdot 6$ & 0.3 & $10 \cdot 8$ & 0.3 & $10 \cdot 8$ & 0.2 \\
\hline Mannose & 1.6 & $<0.1$ & 1.7 & $<0.1$ & 1.7 & $<0.1$ & 1.9 & $<0.1$ & 1.5 & $0 \cdot 1$ & 1.5 & $0 \cdot 1$ \\
\hline Galactose & 4.2 & 0.2 & 3.6 & $<0.1$ & 3.7 & 0.1 & $5 \cdot 1$ & $<0.1$ & 3.6 & 0.2 & 3.9 & $0 \cdot 1$ \\
\hline Glucose & $19 \cdot 0$ & 0.9 & $21 \cdot 2$ & 0.2 & $20 \cdot 9$ & 0.3 & $24 \cdot 3$ & 0.6 & $22 \cdot 2$ & $1 \cdot 1$ & $21 \cdot 7$ & 0.7 \\
\hline Galacturonic acid & $23 \cdot 2$ & $2 \cdot 0$ & 28.8 & 0.6 & $30 \cdot 1$ & 1.5 & 24.5 & 0.6 & $27 \cdot 1$ & 1.8 & $26 \cdot 3$ & $1 \cdot 2$ \\
\hline
\end{tabular}

lipid coalescence. These lipid droplets were approximately 6-8 $\mu \mathrm{m}$ in diameter (Fig. 3(f), arrowheads). Lipid distribution in cells from raw almond blocks that had experienced gastric and duodenal digestion fell into two categories after Nile Blue staining (Fig. 3(g)) or in bright field (Fig. 3(h)). Some cells originating from the centre of the blocks contained oleosomes as found in the untreated raw almonds, other cells from the peripheral layers of the blocks contained several large masses of coalesced lipid (Fig. 3(h), arrowheads). At high magnification, many of these lipid masses showed distorted shapes (Fig. 3(i), arrowheads) characteristic of lipid digestion $^{(10)}$. The only lipid-free cells observed were those from the surface layer of the blocks that were cut open during block preparation, confirming the importance of using sharp blades rather than scalpel blades to dissect almond tissue.
The preparation method proved to be a very practical way of establishing the distribution of lipid, and penetration and effect of digestive enzymes within a large population of cells in almond tissue using bright-field microscopy. An added advantage is that many samples in an experimental run can be preserved for microscopy without fixation or freezing as CDTA prevents microbial growth.

Lipid distribution in chewed raw and roasted and digested almonds. The experiment was repeated using the chewed NA and RA samples, before and after digestion (Fig. 4). Using this method, we demonstrated that mastication of NA samples did not result in lipid coalescence (Fig. 4(a)) in the majority of undamaged cells, whereas roasting appeared to rupture the oleosome membrane resulting in coalescence in all cells (Fig. 4(d)). All chewed samples were characterised by
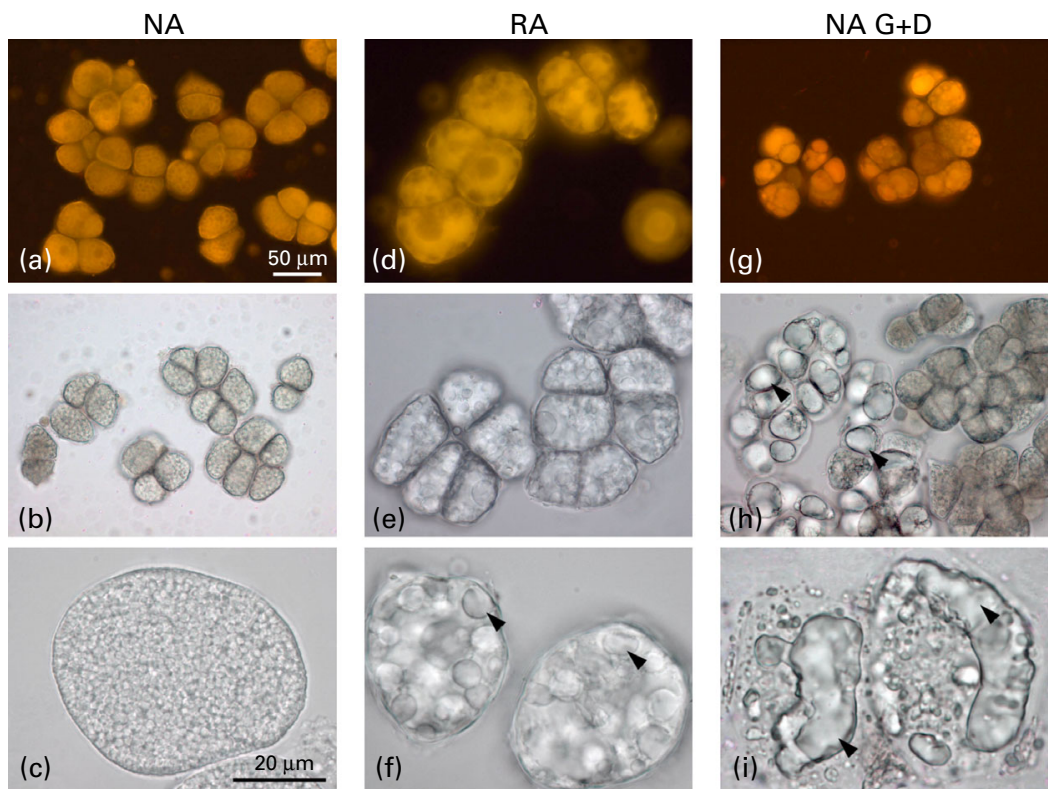

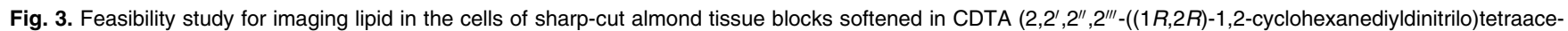
tic acid). In all the cells of raw tissue (NA), lipid is distributed evenly as observed by Nile Blue fluorescence staining (a) and bright field (b), and is located in oleosomes (c). In roasted tissue (RA), lipid is distributed unevenly in all cells as seen after Nile Blue staining (d) and in bright field (e), having coalesced into larger drops (f) (arrowheads). In digested raw tissue (NA G + D), lipid distribution varies between the cells, those from the centre of the blocks contain oleosomes ( $g$, h), those from the outer layers of the blocks contain large lipid masses (h) (arrowheads) that are often irregular in outline (i) (arrowheads). Scale bar (a) applies to all except (c), (f) and (i). For the latter, the scale bar in (c) applies. A colour version of this figure can be found online at http://www.journals.cambridge.org/bjn 

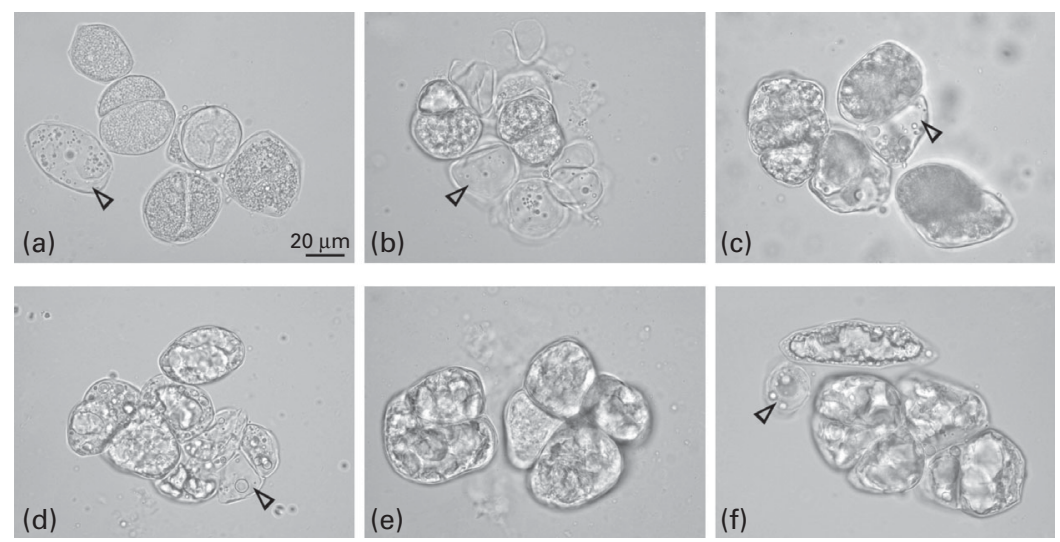

Fig. 4. Bright-field images of chewed raw, roasted and digested almond cells separated by CDTA $\left(2,2^{\prime}, 2^{\prime \prime}, 2^{\prime \prime \prime}-((1 R, 2 R)-1,2-c y c l o h e x a n e d i y l d i n i t r i l o) t e t r a a c e t i c\right.$ acid). (a) Lipid in natural raw almond cells (NA) is present as oleosomes but some cells damaged by chewing contain very little lipid (arrowhead). (b) In NA G (natural raw cells after in vitro gastric digestion), some lipid coalescence has occurred and there are empty cells (arrowhead). (c) In NA G+D (natural raw cells after in vitro gastric + duodenal digestion), there are cells with oleosomes, cells with large lipid aggregates and empty cells (arrowhead). (d) Roasting causes all lipid to coalesce (RA), and tissue becomes brittle resulting in cell damage (arrowhead). (e) Cells of RA G (roasted cells after in vitro gastric digestion) are characteristically either full of coalesced lipid or empty. (f) Cells of RA G+D (roasted cells after in vitro gastric + duodenal digestion) are full of irregular masses typical of lipid digestion or empty cells (arrowhead). Scale bar in (a) applies to all images.

the presence of empty cells (Fig. 4, arrowheads) presumably damaged or crushed sufficiently to release their lipid contents during mastication.

No significant changes were observed after gastric incubation in either NA (Fig. 4(b)) or RA (Fig. 4(e)) samples. After the duodenal phase, cells from the fragments of chewed raw almonds (NA G+D) were either full of oleosomes (from the centre of the samples), full of distorted oil masses in the process of digestion, or empty (Fig. 4(c)). In contrast, cells from the digested roasted material (RA G+D) contained only coalesced or partially digested lipid masses, with many empty cells (Fig. 4(f)).

The conditions present in the DGM, including low $\mathrm{pH}$ and mechanical agitation, did not cause coalescence in all cells, indicating that no endogenous factor was responsible. However, particle size seemed to be an important factor, as most of the enzyme activity was observed in peripheral cell layers.

\section{Discussion}

The results of the present study confirm that the structural integrity of almond cell walls plays a crucial role in regulating lipid bioaccessibility, as assessed using a dynamic gut model that simulates lipid digestion in the gut, and includes, for the first time, a human mastication stage. These findings, combined with our previous in vitro and in vivo observations, provide compelling evidence to indicate that the physical encapsulation of intracellular lipid by the cell walls significantly reduces the rate and extent of lipid release and digestion from almonds in the upper gastrointestinal tract ${ }^{(9,10)}$

However, our observation that a considerable further loss of lipid was observed during the duodenal phase (32.2 and $32.7 \%$ for the NA and RA samples, respectively) over that detected in the gastric environment (16.4 and 15.9\% for the NA and RA samples, respectively) contrasts with our previous findings ${ }^{(10)}$. Our previous study, which focused on sharp-cut almond cubes and finely ground almonds, showed that gastric + duodenal digestion produced only a slight increase in lipid release over and above that of the gastric phase alone. One possible reason for the difference could be that in the present study, the dynamic model of in vitro gastric digestion (DGM) was used. In this model, the digestion products are removed during the time course of the experiment in order to prevent product inhibition. However, we believe that the differences are more likely due to the type and extent of tissue fracture and cell rupture during controlled cutting with a sharp blade compared with the crushing action of mastication. In Mandalari et al. $^{(10)}$, sharp-cut cubes experienced cellular fracture only along the cutting planes, with very little damage beneath (see Fig. $3^{(10)}$ ). Lipid from the ruptured cells at the cut surfaces would be readily bioaccessible, and thus liberated into the lumen during the early gastric phase of digestion. Lipids remaining within the almond cubes would be encapsulated by the cell walls and thus retained during the subsequent duodenal phase - hence negligible lipid was released. However, in the present study, it would be expected that the crushing action of mastication would cause deep fractures into the tissue, creating fissures for diffusion of digestive agents into the tissue, and release of lipids from within to form a lipid barrier around the fragments. During gastric digestion, phase separation between the lipid and aqueous components would have minimised the release of lipid from within the cracked tissues. However, during the duodenal phase, the ingress of bile salts and lipases would facilitate the emulsification and digestion of a significant proportion of the remaining lipid, enabling it to diffuse into the surrounding medium. A proportion of cells remained intact and filled with lipid (as demonstrated by microscopy of the CDTA-separated cells), thereby preventing bioaccessibility to about one-third of the total lipid. Mastication, in effect, increases the surface area of ruptured cells, and the impact of this on lipid digestion is manifest in the duodenum. These observations and hypothesis may help in understanding the importance of cell-wall encapsulation from a metabolic 
perspective, which was highlighted in a recent study, confirming that intact cell walls significantly reduced postprandial lipaemia ${ }^{(11)}$.

The delivery of plant tissue material, comprising intact cell walls (dietary fibre) and undigested intracellular nutrients, including encapsulated lipid and protein, to the large intestine has important implications for energy metabolism and behaviour of the gut microflora. Thus, our previous pilot ileostomy study has shown that a large proportion of the lipid in almonds (i.e. 57-60\% of the total lipid content) remains undigested at the end of the terminal ileum and is fermented by the gut microbiota in the large bowel ${ }^{(10,18)}$. Moreover, unbroken almond parenchyma cells containing intact oleosomes were identified by microscopy in faecal samples from subjects fed an almond-rich diet. Direct analysis of the faeces showed an increase in fat excretion after almond consumption ${ }^{(9)}$. These findings are consistent with evidence showing a strong inverse association between the frequency of nut consumption and BMI and negligible changes in body weight after regular intake of nuts in free-living adults ${ }^{(19)}$.

Another important factor related to energy balance is the role played by mastication and the particle size distribution in the bolus. It has been postulated that mastication affects bioaccessibility and satiety: prolonged mastication could result in higher lipid release, digestion and increased energy absorption $^{(20)}$.

Although roasting had an effect on particle size distribution after mastication, no significant differences in lipid release were observed between the NA and RA samples in either the stomach or the small intestine. A small increase in lipid digestion occurred in the stomach over that observed after oral processing for both raw and roasted almonds, suggesting a fast solubilisation from cells ruptured during mastication, whereas much of the intracellular lipid is probably retained within the cell-wall barrier in intact cells. The presence of pancreatic lipase and surfactants such as bile salts initiated a further increase in lipid release in the duodenum, presumably related to lipid that was encapsulated and became available either through lipase penetration through the cell-wall network into the cells or the entry through small-scale cracks from the peripheral surface of the almond particles into the underlying cell layers. In a control set of experiments performed without addition of enzymes, no increase in lipid release was detected in the duodenal compartment (data not shown).

Microscopy confirmed that the structure of oleosomes, in most cells of raw almonds did not appear significantly altered after mastication, although it was clear that some coalescence occurred as a result of chewing. Roasting caused partial coalescence into larger droplets, but very little change in lipid distribution was noted in raw or roasted tissue after in vitro gastric digestion. However, significant changes were observed in both samples after gastric-duodenal incubation, with the formation of large irregular lipid masses typical of digestion, and lipid loss from damaged cells.

Consistent with our previous investigation ${ }^{(10)}$, there were no changes in the chemical composition of almond cell walls before and after digestion, with the exception of a slight increase in galacturonic acid in the raw samples after gastric + duodenal digestion. Our previous study showed significant cell-wall swelling, which may have contributed to an increased porosity to digestive fluids, including lipase, and thus increased the likelihood of digestion of lipid inside intact cells. Cell-wall swelling could not be followed in the present study because CDTA that is used to separate cells chelates Ca-linked pectins, and thus may alter cell-wall dimensions.

In summary, the results of the present study indicate that processing, such as roasting, had a small effect on particle size distribution after oral processing, which was not significant in terms of lipid release in both the gastric and duodenal environments. The cell walls act as barriers to prevent the physical release of lipid from intact cells. Further studies are needed to investigate the mechanisms by which micronutrient absorption may be affected by encapsulation within cell walls.

\section{Acknowledgements}

The authors gratefully acknowledge the Almond Board of California (CA, USA) for providing the almond samples and Jack Dainty for assistance in the statistical analyses.

The present study was funded by the BBSRC DRINC grants $\mathrm{BB} / \mathrm{H} 004866 / 1$ and $\mathrm{BB} / \mathrm{H} 004874 / 1$ and the University of Messina.

The contributions of each author are as follows: G. M., M. M.-L. G., T. G., C. B., P. J. B., R. M. F., P. J. W., P. R. E. and K. W. W. designed the research; G. M., M. M.-L. G., T. G., M. L. P., K. L. C. and S. C. conducted the research; G. M., M. M.-L. G., T. G., M. L. P., K. L. C., D. B., E. B. and G. L. analysed the data and samples; G. M., M. M.-L. G., T. G., M. L. P., R. M. F., P. R. E. and K. W. W. wrote the manuscript; K. W. W. took primary responsibility for the final content of the manuscript. All authors read and approved the final manuscript.

The authors declare that there are no conflicts of interest.

\section{References}

1. Cohen AE \& Johnston CS (2011) Almond ingestion at mealtime reduces postprandial glycemia and chronic ingestion reduces haemoglobin A1c in individuals with well-controlled type 2 diabetes mellitus. Metabolism 60, 1312-1317.

2. Li SC, Liu YH, Liu JF, et al. (2011) Almond consumption improved glycemic control and lipid profiles in patients with type 2 diabetes mellitus. Metabolism 60, 474-479.

3. Fraser GE, Bennett HW, Jaceldo KB, et al. (2002) Effect on body weight of a free 76 kilojoule (320 kilocalories) daily supplement of almonds for six months. J Am Coll Nutr 21, $275-283$.

4. Scott LW, Balasubramanyam A, Kimball KT, et al. (2003) Long-term, randomized clinical trial of two diets in the metabolic syndrome and type 2 diabetes. Diabetes Care 26, 2481-2482.

5. Wien M, Sabaté J, Ikle D, et al. (2003) Almonds vs complex carbohydrates in a weight reduction program. Int J Obes $\mathbf{2 7}$, 1365-1372.

6. Hollis J \& Mattes R (2007) Effect of chronic consumption of almonds on body weight in healthy humans. BrJ Nutr $\mathbf{9 8}$, 651-656. 
7. Novotny JA, Gebauer SK \& Baer DJ (2012) Discrepancy between the Atwater factor predicted and empirically measured energy values of almonds in human diets. $\mathrm{Am} \mathrm{J}$ Clin Nutr 96, 296-301.

8. Cassady BA, Hollis JH, Fulford AD, et al. (2009) Mastication of almonds: effects of lipid bioaccessibility, appetite, and hormone response. Am J Clin Nutr 89, 794-800.

9. Ellis PR, Kendall CWC, Ren Y, et al. (2004) Role of cell walls in the bioaccessibility of lipids in almond seeds. Am J Clin Nutr 80, 604-613.

10. Mandalari G, Faulks RM, Rich GT, et al. (2008) Release of protein, lipid, and vitamin $\mathrm{E}$ from almond seeds during digestion. J Agric Food Chem 56, 3409-3416.

11. Berry SE, Tydeman EA, Lewis HB, et al. (2008) Manipulation of lipid bioaccessibility of almond seeds influences postprandial lipemia in healthy human subjects. Am J Clin Nutr 88, 922-929.

12. Vardakou M, Mercuri A, Barker SA, et al. (2011) Achieving antral grinding forces in biorelevant in vitro models: comparing the USP dissolution apparatus II and the dynamic gastric model with human in vivo data. AAPS PharmSciTech 12, 620-626.

13. Pitino I, Randazzo CL, Mandalari G, et al. (2010) Survival of Lactobacillus rhamnosus strains in the upper gastrointestinal tract: static and dynamic digestion systems. Food Microbiol 27, 1121-1127.

14. Parker ML \& Guerra W (2008) Occurrence and implications for postharvest quality of intercellular callus hair growth in the outer cortex of apples of "Fuji" and "Fuji' sports. Postharv Biol Technol 48, 192-198.

15. AOAC (Association of Official Analytical Chemists) (1995) Official Methods of Analysis, 16th ed. Gaithersburg, MD: AOAC International.

16. Blakeney AB, Harris PJ \& Stone BAA (1983) A simple and rapid preparation of alditol acetates for monosaccharides analysis. Carbohydr Res 113, 291-299.

17. Blumenkrantz N \& Asboe-Hansen G (1973) New method for quantitative determination of uronic acids. Anal Biochem 54, 484-489.

18. Mandalari G, Nueno-Palop C, Bisignano G, et al. (2008) Potential prebiotic properties of almond (Amygdalus communis L.) seeds. Appl Environ Microbiol 74, 4264-4270.

19. Alper CM \& Mattes RD (2002) Effects of chronic peanut consumption on energy balance and hedonics. Int $J$ Obes Relat Metab Disord 26, 1129-1137.

20. Frecka JM, Hollis JH \& Mattes RD (2008) Effect of appetite, BMI, food form and flavour on mastication: almonds as a test food. Eur J Clin Nutr 62, 1231-1238. 